\title{
Consumos de riesgo: menores y juegos de azar online. El problema del "juego responsable"
}

\author{
Pablo García RuIZ \\ Universidad de Zaragoza \\ pgruiz@unizar.es
}

\author{
Pilar BuIL \\ Universitat Internacional de Catalunya \\ pbuil@uic.es \\ María José SolÉ Moratilla \\ Consell de l'Audiovisual de Catalunya \\ mariajosesole@gmail.com
}

Recibido: 27-01-2015

Aceptado: 17-11-2015

\section{Resumen}

Los juegos de azar online son una actividad muy popular entre los adolescentes. Sin embargo, desde hace un tiempo se ha observado un incremento notable del número de jóvenes que sufren o están al borde de la ludopatía. En este artículo revisamos el impacto de estas prácticas sobre los jóvenes y discutimos la conveniencia de promover un concepto de "juego responsable" que permita alertar de sus riesgos e impida eficazmente el acceso a los menores de edad. Los principales factores asociados al juego problemático son la edad de inicio, el entorno familiar, la influencia de la publicidad, el consumo de sustancias estimulantes y las actitudes del grupo de iguales. Los poderes públicos y la misma industria del juego deben considerar estos factores y concretar el desarrollo de planes que promuevan un modelo de juego seguro y controlado. La publicidad debe tener en cuenta criterios de protección de los consumidores sabiendo que, aun cuando les está prohibido, los menores acceden con facilidad al juego de azar online. Las Administraciones Públicas deben establecer políticas y sensibilizar a la sociedad. Los agentes sociales implicados deben aportar mecanismos de prevención y sensibilización hacia un problema del que existe aún poca conciencia social y una excesiva despreocupación.

Palabras clave: consumo; riesgo; juegos de azar online; internet; menores; adicción; responsabilidad social corporativa; publicidad.

\section{Risk Consumption: Children and Online Gaming. The Problem of "Responsible Gambling"}

\begin{abstract}
Online gambling is a popular activity among adolescents. However, there has been a notable increase in the number of young people who suffer or are on the verge of pathological gambling. We review the impact of online gambling on young people and discuss the desiderability of the concept of "gambling
\end{abstract}


responsibly" in order to alert of their risks and effectively prevent access to minors. The main factors associated with pathological gambling are the age of start, the family environment, the influence of advertising, the consumption of stimulants, and the attitudes of the peer group. Both the government and the gaming industry itself should consider these factors and develop comprehensive plans that ensure a safe and controlled model game. In this context, advertising must take into account criteria of consumer protection knowing that even if they are not allowed, children can easily have access to online gambling. All agents involved, including public and social agents, must provide mechanisms for prevention and awareness of a problem that arouses little social consciousness and excessive carelessness.

Key words: risk; consumption; online gambling; internet; children, addiction; corporate social responsibility; advertising.

\section{Referencia normalizada}

García Ruiz P., P. Buil y M.J. Solé Moratilla (2016): “Consumo de riesgo: menores y juegos de azar online. El problema del "juego responsable"”, Política y Sociedad, 53 (2), pp. 551-575.

Sumario: Introducción. 1. El impacto de los juegos de azar en adolescentes. 2. La ludopatía entre adolescentes. 3. Políticas de protección y prevención. 4. El concepto de "juego responsable". 5. Conclusiones. 6. Bibliografía.

\section{Introducción}

Los juegos de azar online son una actividad muy popular entre los adolescentes. Sin embargo, desde hace un tiempo se ha observado un incremento notable del número de jóvenes que sufren o están al borde de la ludopatía (Kuss y Griffiths, 2012; MuñozMolina, 2008; Petit et al., 2015; Scholes-Balog et al., 2014; Splevins et al., 2010). En torno a otros "consumos de riesgo", como el de tabaco y alcohol, se han generado amplios consensos sobre la necesidad de alertar de los daños asociados y prohibir el acceso a los menores de edad, promoviendo así un "consumo responsable". La Comisión Europea (2014) ha exhortado a los Estados miembros a promover medidas que minimicen los riesgos derivados de los juegos de azar online. El propósito general es preservar la salud y elevar el nivel de protección de todos los ciudadanos, en particular los menores, frente a los riesgos que puedan derivarse de una participación excesiva en las apuestas online. En este artículo examinamos la influencia de estas prácticas sobre los jóvenes y discutimos la conveniencia de promover un concepto adecuado de "juego responsable".

\section{El impacto de los juegos de azar en adolescentes}

Para la generación actual de adolescentes los medios de comunicación contribuyen de forma decisiva a la creación de un contexto social en el que surgen nuevas relaciones e interacciones comunicativas (Fernández Cavia, 2009). Ellos han nacido y crecido en medio de pantallas y conviven con múltiples dispositivos a su alrededor que encauzan la interacción en las redes sociales a la vez que albergan contenidos específicos, de carácter comercial y lúdico (Duek, 2010). Las actividades de ocio de los adolescentes incluyen muy diversos tipos de juegos online cuyo éxito e importancia se ha difundido 
ampliamente en los últimos años (Kopp, 2015). Entre ellos, ocupan un lugar destacado los juegos de azar.

Los juegos de azar son juegos en los cuales la posibilidad de ganar o perder está condicionada de forma decisiva por el azar, de modo que no dependen exclusivamente de la habilidad del jugador. En algunos tipos de juegos de azar como son las loterías, el bingo y la mayoría de las máquinas tragaperras, los resultados son aleatorios e impredecibles. En cambio, hay otros juegos de azar, como son las apuestas deportivas, en los que el uso de habilidades y conocimientos puede dar una ventaja sobre otros jugadores, de forma que aquí sí que habría una cierta previsibilidad en los resultados (Griffiths, 2009). Además, quienes participan suelen apostar dinero o bienes valiosos. El juego de azar tiene una dimensión económica que se refiere a correr el riesgo -eso significa apostar- de ganar o perder en función del resultado. El éxito o fracaso posible supone un potente valor motivacional. El término juego online incluye exclusivamente los juegos de azar online en los que concurren el azar y el riesgo económico, a los se accede mediante una plataforma operativa en Internet.

La mayoría de los estudios sobre los hábitos de juego de los adolescentes se han realizado en Estados Unidos, Canadá, Reino Unido y Australia (Griffiths, 2001; Wiebe y Falkowsky-Ham, 2003; Griffiths et al., 2009; Ipsos MORI, 2009; Splevins et al., 2010; Brunelle et al., 2012; McBride y Derevensky, 2012; Parker et al., 2013), si bien en los últimos años se han llevado a cabo estudios también en diversos países de Europa, como los de Raisamo et al., (2012) en Finlandia; Tsitsika et al., (2011) y Floros et al., (2012) en Grecia, Olason et al., (2011) en Islandia, Erbas y Buchner (2012) en Alemania y Cosenza y Nigro (2015) en Italia. En España, se han realizado algunos estudios sobre la incidencia del juego entre la población en general (Gómez-Yañez, 2015; Jiménez-Murcia et al., 2014) pero pocos sobre el juego entre adolescentes. El estudio más reciente sobre adolescentes es el publicado por Carbonell y Montiel (2013) que reúne una parte cuantitativa y otra cualitativa. La primera consiste en una encuesta a 4.126 adolescentes (52,8\% chicas) de la Comunidad Valenciana, de 12 a 20 años, estudiantes de Secundaria y Bachillerato. La segunda, en cuatro grupos de discusión en los que participaron adolecentes valencianos (19 chicos y 18 chicas) de 12 a 17 años, también estudiantes de Secundaria y Bachillerato. En todos estos estudios apoyamos el análisis sobre el impacto de los juegos de azar que exponemos en las siguientes páginas, indicando el universo concreto al que se refieren cuando así resulte necesario. También nos basamos en importantes trabajos de revisión y síntesis como los de Fröberg (2006), Valentine (2008), Griffiths y Parke (2010), Volberg et al., (2010), Kuss y Griffiths (2012) y Derevensky y Gilbeau (2015), que aportan una perspectiva internacional comparada y permiten algunas generalizaciones sobre un referente tan variado y multidimensional como es el de los adolescentes y su relación con los juegos de azar online.

Entre la juventud, el uso de los juegos de azar es un hecho que va en aumento. Aunque los juegos de azar se han visto tradicionalmente como una actividad de adultos, hay amplia evidencia sobre su popularidad entre adolescentes que se implican en diversos juegos de azar regulados (a pesar de que están prohibidos para los menores de 18 años) y no regulados. Este hecho es, probablemente, consecuencia de la creciente aceptación social de los juegos de azar, la abundante publicidad que lo promociona, la asociación 
con personajes famosos y la aprobación por parte de los gobiernos, junto al glamour asociado con los casinos y popularizado por películas de Hollywood como Ocean's Eleven o Casino Royale (Derevensky, 2012; Volberg et al., 2010). Se puede afirmar que la actual generación de adolescentes ha sido la primera que ha crecido en una sociedad en la que los juegos de azar están ampliamente aceptados y son fácilmente reconocidos como un entretenimiento, si bien en algunos países, como los anglosajones, esto ha ocurrido antes y más ampliamente que en otros (Derevensky y Gilbeau, 2015).

Los juegos más populares entre los adolescentes incluyen los juegos de cartas, las apuestas deportivas, los dados y los juegos de mesa con familiares y amigos; también las apuestas en grupos de iguales sobre juegos de habilidad personal (por ejemplo, los bolos, las carreras, etc.), la competición en videojuegos con premio en metálico, la adquisición de boletos de lotería, el juego en las máquinas tragaperras (slot machines), las apuestas en las carreras de caballos (Grifftihs y Parke, 2010; Kuss y Griffiths, 2012). En los últimos años, con la facilidad de acceso que supone el juego por Internet, se ha observado un número creciente de adolescentes que participan en esos juegos desde su ordenador o su smartphone, y que también por esta vía participan en otros juegos reservados para mayores de 18 años, como los juegos de casino, las video lottery terminals, o los concursos de poker, entre otros (McBride y Derevensky, 2012; Derevensky y Gilbeau, 2015).

Los avances tecnológicos han hecho que los juegos de azar estén evolucionando y adaptándose a un nuevo entorno. Los juegos de azar online apelan a las habilidades tecnológicas de los jóvenes, al buscar la convergencia entre los juegos de habilidad y los juegos de azar (Griffiths, 2009). Además, la escasa capacidad de las web de juego para comprobar la edad de los participantes permite que los adolescentes participen en nuevos juegos más atractivos que con frecuencia perciben como un reto para sus capacidades personales. Las redes sociales se han convertido en uno de los cauces de acceso a los juegos de azar más populares entre los adolescentes (King et al., 2014).

En el estudio de Carbonell y Montiel (2013), los participantes en grupos de discusión compuestos por adolescentes de entre 12 y 17 años manifestaron conocer al menos 15 páginas web que ofrecen la posibilidad de acceder a juegos de azar online. Entre ellas, la más conocida es Facebook. La mayoría de los adolecentes conocía y utilizaba el sitio web Tuenti -la red social española con más de trece millones de usuarios- para acceder a estos juegos.

Los juegos de azar han llegado a ser una forma más de entretenimiento entre los adolescentes. Wiebe y Falkowsky-Ham (2003) se propusieron estudiar el perfil de los adolescentes que participan en juegos de azar en Canadá. Para ello, encuestaron telefónicamente a 501 adolescentes de 11 a 16 años, incluyendo chicos $(51,2 \%)$ y chicas $(48,8 \%)$. El 75\% había participado durante el año anterior en algún juego de azar. El $9,2 \%$ lo hizo en Internet. La participación va en aumento con la edad. Uno de cada cuatro de todos ellos dijeron que apostar es atractivo (cool). Los que se definen a sí mismos como personas populares, amantes del riesgo o líderes juegan en mayor proporción. Los chicos se sienten empujados por sus amigos a jugar con más probabilidad que las chicas ( $24 \%$ frente a $16 \%$ ). Consideran que participar en juegos de azar está bien siempre que uno se atenga a la cantidad que uno se ha fijado ( $89 \%$ de los chicos frente a un $79 \%$ 
de las chicas). Gendron y sus colegas (2009) llevaron a cabo un estudio con 1.876 estudiantes de enseñanza secundaria en Canadá, de entre 14 y 18 años (46\% chicos y $54 \%$ chicas). El 93,5\% de total dijo que había participado en algún juego de azar en los últimos doce meses y el $8 \%$ del total (13\% de los chicos y $3 \%$ de las chicas) dijo que había jugado online en el mismo periodo de tiempo. Olason y sus colaboradores (2011) encuestaron a 1.537 adolescentes islandeses (768 chicos y 747 chicas) de entre 13 y 18 años. Los cuestionarios incluían preguntas sobre la participación en juegos de azar online disponibles en sitios web nacionales (lotto, apuestas deportivas) y en sitios web internacionales (poker, casino, apuestas deportivas y apuestas 'gratis'). El $57 \%$ de los encuestados había jugado alguna vez, el 8\% jugaban regularmente, el 24\% habían jugado alguna vez online y algo más del 4\% jugaban online habitualmente. Griffiths y Parke (2010) concluyen, con base en su amplia revisión de la literatura que un porcentaje pequeño pero significativo de adolescentes participa en juegos de azar online: los datos varían, en función de la edad y el contexto cultural, entre un $2 \%$ y un $8 \%$ si bien la mayoría de los estudios informan de un porcentaje de participación de alrededor de un 4\%. Los datos se incrementan con la edad de los encuestados y resultan más elevados en Reino Unido y Quebec y algo menores en Estados Unidos y Australia.

La participación en los juegos de azar entre adolescentes puede verse como un continuo entre la participación social, ocasional, recreativa, y la implicación excesiva hasta el punto de convertirse en una actividad compulsiva, patológica o "desordenada" (este último es el término escogido por la DSM-5 para referirse al juego seriamente problemático). En el estudio de Wiebe y Falkowsky-Ham (2003) casi un 4\% de los adolescentes de 11-16 años decían haber sufrido problemas relacionados con el juego como discusiones, peleas o pérdidas económicas. Hasta un $16 \%$ indicaba que algunos de sus amigos han sufrido este tipo de problemas. Gendron et al., (2009) informan que el 3\% del total de los adolescentes participantes en su estudio sufrieron problemas con relación al juego. La probabilidad de ser jugadores problemáticos se elevaba al $11 \%$ entre los jugadores online. Olason et al., (2011) infoman de que la prevalencia de juego problemático entre los adolescentes de su estudio era del 3\%. Sin embargo, entre aquellos que habían jugado online, el índice de prevalencia del juego problemático era significativamente más elevado, hasta alcanzar un 7,5\%. Parece, pues, que existe una relación más intensa entre aquellos jóvenes que juegan en Internet y el desarrollo de problemas con el juego.

Los adolescentes se inician en los juegos de azar online a una edad temprana, entre los 10 y los 13 años (Gupta y Derevensky, 2014; EUnet, 2012). Algunos juegan rodeados por un clima familiar, social y recreativo en el que son bienvenidos como jugadores por los propios miembros de la familia o amigos mayores de la propia comunidad (Vander Bilt y Franklin, 2003). En España, donde la práctica de las apuestas es menos aceptada socialmente que en otros países, según los datos recogidos en Carbonell y Montiel (2013), el porcentaje de niños y jóvenes entre 12 y 20 años que juegan en Internet se sitúa en torno al 18\%. Aunque la mayoría de los adolescentes juegan ocasionalmente y no experimentan problema alguno, la investigación académica sugiere que los adolescentes como grupo constituyen una población de riesgo respecto al juego problemático (Derevensky y Gilbeau, 2015). Volberg et al., (2010) apuntan 
que los estudios de prevalencia muestran que entre un $2 \%$ y un $8 \%$ de los adolescentes sufren problemas con el juego y que otro $10-15 \%$ están en riesgo de desarrollarlos (la variabilidad se atribuye a la metodología empleada y a los diversos contextos socioculturales de los universos observados). En algunos países se consideraba ya hace años el juego adolescente como un problema grave de salud pública (Messerlian et al., 2005), pues el porcentaje de los chicos jugadores entre 15 y 17 años que decía jugar lo suficiente como para ser clasificado como jugador patológico o problemático, era muy superior al de los adultos (Fröberg, 2006). Proporciones parecidas, entre el 3,5 y 5\% de jugadores patológicos adolescentes se han encontrado también en España (Arbinaga, 2000; Muñoz-Molina, 2008; Becoña et al., 2001; Carbonell y Montiel, 2013).

\subsection{Los motivos para jugar}

Los adolescentes comienzan a jugar movidos por una serie de razones que van desde las ganancias posibles al placer, el contexto social y el escapismo. Para muchos menores, jugar es un medio para escapar al control parental y mantener una relación de igualdad con otros adolescentes (Fröberg, 2006). Las apuestas online añaden a los juegos de azar presenciales el potencial de ofrecer visualmente excitantes efectos similares a los videojuegos, máquinas tragaperras y VLTs (video lottery terminals) en los que la frecuencia es muy rápida. Todos estos elementos son muy apreciados por los adolescentes (Griffiths y Wood, 2000). La relación de los adolescentes con Internet al igual que sucede con los adultos es intensa. Internet proporciona una realidad alternativa a la presencial y permite la sensación de inmersión y de anonimato que para muchos adolescentes es atractiva y psicológicamente gratificante (Livingstone y Moira, 2013). Los adolescentes perciben los juegos de azar online como una actividad "de riesgo", prácticamente igual de arriesgada que aceptar solicitudes de amistad online de personas desconocidas, o que inventar una identidad para gastar bromas o acceder a contenidos sexuales para adultos (Carbonell y Montiel, 2013: 109). Los jóvenes poseen unos mayores conocimientos informáticos y por su falta de experiencia e inmadurez son susceptibles a los poderes adictivos del juego por Internet por encima de otros grupos de edad.

Entre los factores que inciden en la disposición a jugar (o seguir jugando) se cuentan la frecuencia de los eventos sobre los que apostar, la cantidad del bote, el número de situaciones cercanas a ganar, los efectos de luces y colores, los efectos de sonido, la accesibilidad, la publicidad, las reglas del juego, etc. (Kuss y Griffiths, 2012). También afectan la privacidad, los buenos resultados, la alta velocidad del juego, la fiabilidad de los pagos, el dinero ganado de prima, el reclamo sexual, los gráficos y el anonimato (Deverinsky y Gupta, 2005).

En cuanto al género, los hábitos de juego de chicos y chicas son diferentes al igual que las consecuencias emocionales derivadas de la práctica de juegos de azar (ScholesBalog et al., 2014; Donati et al., 2013; Floros et al., 2012). Los chicos comienzan a jugar a una edad más temprana y, posteriormente, juegan más a menudo y durante períodos más largos que las chicas. En general, los chicos tienden a apostar en juegos de mayor riesgo, como son las apuestas deportivas. Los varones tienden a participar en diferentes tipos de juegos de azar, juegan más a menudo, gastan más tiempo y más dinero, y tienen 
más problemas derivados del juego que las chicas. Una mayor proporción de jugadores con problemas o jugadores patológicos son varones, de dos a cinco veces más. Esto puede explicarse en parte por el hecho de que los chicos juegan más que las chicas y tienden a elegir los juegos de mayor riesgo, lo que a su vez incrementa el riesgo de ludopatías.

Las motivaciones que subyacen al juego -y a continuar jugando a pesar de incurrir en pérdidas repetidas- son muy variadas. En cualquier caso, es interesante advertir que ganar dinero no es típicamente la principal motivación que señalan los adolescentes (Shead et al., 2012). Diversos estudios entre jugadores problemáticos sugieren que la primera motivación para seguir participando en juegos de azar tiene que ver con la excitación (la subida de adrenalina) y el placer que lleva consigo (Derevensky et al., 2011). También se ha apuntado como motivos por los que los jóvenes se implican en los juegos de azar, el paliar el aburrimiento y la soledad, escapar del estrés provocado por tensiones académicas o familiares, reducir la ansiedad, o también emplear el juego como forma de socialización o, incluso, de competición con amigos y compañeros (Shead et al., 2012; Felsher et al., 2010). En el estudio de Wiebe y Falkowski-Ham (2003: 52) los adolescentes canadienses encuestados atribuían diferentes significados a los términos "apuesta" y "juego de azar" y, de hecho, preferían utilizar la palabra "apuesta" para definir las actividades que ellos realizan. Para ellos, la expresión "juegos de azar" tenía un componente negativo, de pérdida de dinero, de meterse en problemas $\mathrm{y}$, en cambio, veían las apuestas como algo menos dañino o problemático.

\subsection{El gancho: webs de juego sin dinero}

La mayoría de sitios de juegos de azar permiten y animan a jugar sin dinero a modo de práctica o de prueba. Los expertos consideran estas webs como inicializadoras de la práctica del juego. Al animar a jugar sin dinero no pesa en los jugadores la responsabilidad de perderlo, pero una vez han probado el juego se sienten animados a arriesgar y entonces es cuando apuestan dinero. Esto también ocurre con los menores de edad, que comienzan a jugar en estos sitos. No es necesario arriesgar el dinero propio aunque en la web se da información sobre las pérdidas o las ganancias ficticias, en términos monetarios (Griffiths y Parke, 2010). En realidad lo que hacen los adolescentes en estos sitios web es aprender a jugar a juegos de azar de adultos. En las páginas de juego gratuito los adolescentes comienzan a conceptualizar y experimentar los juegos de azar sin las consecuencias de estar perdiendo dinero (Ipsos MORI, 2009). Estas opciones de juego gratis influyen en los adolescentes y en el futuro desarrollo de formas de juego problemático pues las ganancias ficticias reportadas a menudo son más frecuentes que las que de hecho se obtienen cuando se apuesta dinero efectivo. Ello induce con facilidad falsas expectativas en los jugadores de que las ganancias y las pérdidas están equilibradas en los escenarios de juego real.

Los sitios de juego gratuitos crean una disociación entre las acciones y las consecuencias ya que los jugadores no están perdiendo dinero real. Los sitios de apuestas gratis están pensados para generar jugadores que en el futuro jueguen con dinero. Tienen el potencial para ser tan adictivos como las webs en las que se juega con dinero. Son espacios en los que los adolescentes tienen sus primeras experiencias 
con los juegos de azar, ya que permiten desarrollar comportamientos y métodos de jugadores reales. Ello significa que en el momento en que los jugadores se muevan en un ambiente de juego con dinero, sus comportamientos y prácticas con el juego serán muy arraigadas y se reflejará en el entorno de juego efectivo (Messerlian et al., 2004: 4). La industria del juego reconoce esta gran similitud entre juego con o sin dinero ya que la mayoría de los casinos online y casas de apuestas ofrecen bonos a los nuevos jugadores. Estas webs de práctica de juegos de azar sin dinero son indistinguibles de las webs en las que se juega con dinero pero, con frecuencia carecen de sistemas de salvaguarda de acceso a menores y, de ordinario, no incorporan ningún tipo de señales y advertencias (Derevensky y Gilbeau, 2015).

\section{La ludopatía entre adolescentes}

La actividad social de juego, que tiene un cierto papel en el desarrollo cognitivo de los seres humanos, puede convertirse en una adicción. El comportamiento adictivo es un hábito de conducta que incrementa el riesgo de padecer problemas personales y sociales. Los comportamientos adictivos son a menudo experiencias subjetivas de pérdida de control.

Desde 1980 la Sociedad Americana de Psiquiatría reconoce la ludopatía oficialmente como "transtorno psicológico" en el Manual diagnóstico y estadístico de los trastornos mentales (DSM-III). Se entiende que muchos comportamientos pueden ser potencialmente adictivos, incluyendo los juegos de azar, los juegos de ordenador y el ejercicio físico, entre otros (Griffiths, 2002; Parker et al., 2013). El avance de las tecnologías promueve nuevos patrones de comportamiento susceptibles de promover un abuso y una dependencia como son, por ejemplo, navegar por Internet o utilizar un teléfono móvil (Ruiz-Olivares et al., 2010). La adolescencia es una etapa del desarrollo en la cual las personas son especialmente vulnerables (Domínguez, 2009). Los adolescentes son más vulnerables a las consecuencias negativas de los juegos de azar que los adultos y las tasas de prevalencia son particularmente más altas que entre adultos (Granero et al., 2014; Valentine 2008).

La ludopatía es la más característica de las adicciones no tóxicas (Derevensky et al., 2011). Este trastorno se caracteriza porque la persona no es capaz de controlar sus impulsos a jugar, lo que le acarrea una serie de graves consecuencias en la esfera personal, familiar, profesional y social. Según la Organización Mundial de la Salud (1995) la ludopatía consiste en la presencia de frecuentes y reiterados episodios de juegos de apuestas, los cuales dominan la vida del enfermo en perjuicio de sus valores y obligaciones sociales, laborales, materiales y familiares del mismo. Los enfermos describen la presencia de un deseo imperioso e intenso a jugar que es difícil de controlar, junto con ideas e imágenes insistentes del acto del juego y de las circunstancias que lo rodean. En esta patología se suelen encontrar las mismas características que en otras adicciones: dependencia psicológica, incapacidad de mantener la abstinencia, síndrome de abstinencia, preocupación continua por el juego, pérdida de control, deterioro de otras áreas a consecuencia de la conducta de juego (familiar, social, laboral, etc.). El proceso de la dependencia al juego sigue las mismas etapas evolutivas que el resto de 
las dependencias. El inicio suele darse en la adolescencia y con pequeñas apuestas, aunque puede iniciarse a cualquier edad.

\subsection{Variables influyentes y factores de riesgo}

Lo que transforma a un jugador social en un ludópata es la necesidad de huir de una situación problemática (Derevensky, 2012). La necesidad de evadirse de la realidad aparece recurrentemente en los discursos de todos los jugadores enfermos. Se empieza a jugar porque gusta, por la facilidad de acceso. En todos los casos siempre hay un estímulo que facilita que la actividad se vuelva problemática sin que quien la inicia se dé cuenta. Los tres mecanismos de motivación más influyentes en la adicción al juego son: ganar dinero, escapar de los problemas y buscar "activación" (Carpio, 2009: 98). En este sentido, el juego actúa como un estimulante, que ayudaría a conseguir un estado de ánimo eufórico. El riesgo y la excitación como algo placentero en determinadas personas las hace más vulnerables a sufrir una adicción. No hay evidencia empírica de que haya "rasgos" de personalidad específicos en las personas jugadoras; cualquier tipo de personalidad es susceptible de desarrollar una ludopatía, aunque hay veces en que en la adicción subyace un problema de personalidad, de baja autoestima (Carpio, 2009: 31). El jugador patológico se encuentra influido por diferentes factores entre los que destacan la cultura, la sociedad, el trabajo, la familia, el círculo de amigos y la influencia de variables biológicas en el proceso (Domínguez, 2007).

Datos de varios países indican que el juego patológico se inicia a edades tan tempranas como 10 u 11 años (Becoña, 2004; Brunelle et al., 2012; Splevins et al., 2010; Weinberger et al., 2015; Olason et al., 2011). La influencia de los padres es muy importante: si los padres juegan con sus amigos o con sus hijos o compran lotería a los hijos o tarjetas de rascar, los hijos desde pequeños ya desarrollan conceptos de juegos de azar observando a sus padres (Lesieur, 2003; Floros et al., 2012). Los adolescentes con padres que juegan a juegos de azar y que comienzan a jugar a una edad más temprana, son los que más a menudo tienen problemas con el juego en comparación con los adolescentes con padres que no juegan a juegos de azar (Derevenksy y Gilbeau, 2015). Así, hay razones para creer que el juego de los padres está relacionado con los problemas con el juego. Fröberg (2006) en su estudio sobre el juego entre adolecentes suecos puso de manifiesto que entre un $25 \%$ y un $40 \%$ de los padres de adolescentes con problemas de juego eran jugadores patológicos o con otro tipo de adicciones. La existencia de una relación parental pobre se asocia con factores que contribuyen al desarrollo de conductas adictivas. Los estudios han observado que el grupo formado por probables personas jugadoras patológicas jóvenes y el grupo de riesgo de desarrollo de la ludopatía, percibían un apoyo familiar menor (Scholes-Balog et al., 2014). Muchos jóvenes en esta situación percibían a sus progenitores y a otros familiares importantes como poco cariñosos, severos o demasiado críticos, sintiéndose emocionalmente distanciados o distantes de los miembros de la familia.

En el desarrollo de la ludopatía, es importante también la relación con el entorno. Los factores psicológicos ligados a las redes sociales de los adolescentes son importantes porque la vulnerabilidad en un adolescente comporta que "determinadas experiencias pueden tener efecto en el modo en el que van a construir su particular concepción del 
mundo" (Domínguez, 2007: 149). En efecto, los adolescentes pasan gran parte de la jornada en la escuela y allí desarrollan relaciones sociales con el entorno, en la misma medida en que extienden estas relaciones se separan de su familia y de sus padres, es la etapa en la que el adolescente está más receptivo a sentimientos, valores, ideales, actitudes y hábitos de significación ético social. Las actitudes sociales están orientadas a la necesidad de aprobación de conformidad, de reconocimiento y participación. Surgen entonces los clubes, los equipos que favorecen el proceso de socialización. Se adquieren muchos conceptos fundamentales que están en la base de una buena vida social. La tendencia de los adolescentes a dar más importancia al grupo de iguales que a la familia hace pensar a los investigadores que el apoyo de los iguales influye más que el apoyo familiar y eso es importante en relación al uso de sustancias y a la actividad del juego entre adolescentes (McBride y Derevensky, 2012).

Entre las variables sociales que influyen en la adicción al juego son especialmente importantes las siguientes (Carpio, 2009): presión del grupo de iguales, presión de la publicidad, alta permisividad social con el juego de azar, fácil acceso al juego, apuestas asequibles, escaso tiempo entre apuesta y resultado y dispositivos que facilitan la ilusión de control (mover palancas, elegir opciones, etc.). El hecho de jugar solo hace que cada adolescente decida cuánto tiempo y cuánto dinero va a invertir lo cual le proporciona una relativa facilidad para jugar sin que nadie le controle. Esta característica en un adolescente constituye un factor de riesgo (McBride y Derevensky, 2012). También son importantes aquellos valores sociales en los que impera la idea de la cultura de las ganancias fáciles y rápidas, el consumo excesivo y la riqueza como valor supremo de felicidad.

Conviene destacar también que los medios de comunicación que ejercen un poder de persuasión importante sobre los jóvenes (Aguaded-Gómez, 2011). Los juegos de azar se reflejan en la publicidad y en los medios de comunicación no sólo como una forma de entretenimiento con los amigos, sino también como una actividad con la que se puede ganar dinero (McMullan et al., 2012). Cómo influye en los adolescentes la publicidad en los diferentes soportes, es algo que debe ser examinado para poder también identificar medidas preventivas o legales que ayuden a disminuir los índices de prevalencia de menores que participan en juegos de azar.

\subsection{Problemas asociados a los jugadores patológicos adolescentes}

Los juegos de azar, para muchos adolescentes, son parte de un conjunto más amplio de comportamientos de riesgo como el tabaquismo, el alcohol y otras drogas (Weinberger et al., 2015; Barnes et al., 2011). Los adolescentes que son jugadores patológicos padecen también depresión y síntomas de ansiedad con más frecuencia que otros adolescentes (Cosenza y Nigro, 2015; Messerlian et al., 2005). Los adolescentes que tienen problemas con el juego son, también, más propensos a un comportamiento delictivo en comparación con los adolescentes sin problemas con el juego (Brunelle et al., 2012). Los jugadores problemáticos tienen dificultades en la escuela en mayor medida que los demás adolescentes. En su revisión de los estudios disponibles Kuss y Griffiths (2012) muestran unos resultados variables en las relaciones entre el juego y otras conductas excesivas, pero advierten que existe una superposición entre el juego de azar y otras 
conductas de riesgo. Lo que no se puede establecer es que estos comportamientos sean la causa de la participación en juegos de azar por parte de los adolescentes.

Pocos estudios han seguido grupos de jóvenes durante varios años para ver cómo su problema con el juego patológico se ha desarrollado y si los problemas persisten en la edad adulta o si se ha producido una mejora. Según los estudios disponibles los problemas con el juego en adolescentes no parece que sean "estados crónicos". Parece que son estados temporales que se mitigan con la edad (Granero et al., 2014). Según Svensson (2011) los problemas de juego de los adolescentes son más variables que los de los adultos porque los jóvenes son más propensos a asumir riesgos y no suelen limitar su juego.

La prevalencia del juego patológico es particularmente alta entre adolescentes comparada con los adultos, si bien a partir de los 18 años muchos adolescentes dejan de jugar de forma problemática (Granero et al., 2014; Derevensky y Gilbeau, 2015). Los jugadores problemáticos presentan un patrón característico de uso de Internet (inicio temprano, uso intensivo y extensivo), asumen más riesgos online y viven más experiencias indeseadas en la red. Los datos de Carbonell y Montiel (2013) apuntan a que la prevalencia del juego online se ha incrementado notablemente en los últimos años. De todos los encuestados, casi la mitad eran jugadores que juegan siempre que se conectan a Internet, que prefieren conectarse por la noche y les es indiferente el lugar o contexto en el que se encuentren, el medio tecnológico desde donde se conectan y las normas que existan. Entre ellos, la elevada frecuencia de juego está relacionada con un bajo rendimiento académico, así como con una baja consideración de las propias relaciones familiares dentro de un perfil psicológico de elevada vulnerabilidad.

En su revisión de la literatura sobre juego problemático Derevensky y Gilbeau (2015) apuntan que hay más chicos que chicas adolescentes con problemas relativos a los juegos de azar. Los chicos suelen hacer apuestas más elevadas, comienzan a edad más temprana, participan en más actividades y más diversas, juegan con más frecuencia, le dedican más tiempo, gastan más dinero y experimentan más problemas que las chicas (Derevensky, 2012; Donati et al., 2013; Volberg et al., 2010). Los jóvenes con problemas con frecuencia han obtenido alguna ganancia grande al comienzo de su relación con el juego (Griffiths, 2009), han tenido también la influencia de padres o familiares que a su vez son jugadores 'desordenados' y de algunos de sus amigos que también tienen problemas de juego patológico (Griffiths y Parke, 2010). La mayoría de los adolescentes con problemas de juego no reconocen la severidad de su problema y perciben los riesgos asociados con el juego desordenado como algo que puede ocurrir a largo plazo pero no como una preocupación inmediata (Hardoon et al., 2003; Valentine, 2008). Las diferencias culturales y étnicas parecen tener escaso impacto sobre la percepción de la seriedad del problema (Volberg et al., 2010). Los adolescentes con problemas de juego patológico muestran, además, mayores niveles de excitabilidad y ansiedad, impulsividad, propensión al riesgo y desinhibición (Derevensky y Gilbeau, 2015). También manifiestan mayor inicidencia de problemas académicos y conductuales en la escuela, problemas de salud mental y de otro tipo de adicciones. (Barnes et al., 2011). El hecho de que estas conductas y factores de riesgo estén relacionados puede 
apuntar a que el juego problemático se dé en el marco de una conducta problemática más general.

\section{Políticas de protección y prevención}

\subsection{Regulación pública}

Si la actividad de los juegos de azar conlleva riesgos, los Estados deben proveer la forma de prevenir, paliar y sostener las consecuencias negativas derivadas de esta actividad o de su falta de control o de su mala regulación.

Los aspectos que el ordenamiento jurídico debe tener en cuenta son (Dorrego, 2011), por una parte, el cuidado de la salud pública amenazada por los riesgos asociados a la adquisición de adicciones; por otra parte, la protección de los menores y de las demás personas vulnerables que hace necesario el establecimiento de medidas que les impidan participar; además, los poderes públicos deben velar por la protección general de consumidores y usuarios adoptando dispositivos de seguridad que impidan la posibilidad de acciones delictivas. La tutela jurídico-pública del jugador es uno de los objetivos de la regulación y teniendo en cuenta los índices de prevalencia de adultos y menores, es importante analizar si la legislación vigente está sirviendo para proteger el bien jurídico que representa el jugador dependiente.

Las políticas sociales en relación con los juegos de azar han de tener en cuenta, por una parte, los costes sociales y, por otra, las necesidades de protección y prevención de comportamientos patológicos. En efecto, la adicción al juego, aunque afecta fundamentalmente al jugador, también genera costes para la sociedad en su conjunto. El juego patológico destruye a quien lo practica y deteriora el modo de vida de quienes rodean al jugador. Entre los costes sociales del juego podemos destacar los costes económicos relacionados con la ludopatía, que la sociedad y que en última instancia el Estado va a tener que hacer frente. Sin duda, los juegos de azar generan beneficios económicos para el Estado, ya sea por los ingresos procedentes de impuestos o por la creación de puestos de trabajo. Pero tales beneficios llevan asociados diversos tipos de costes no sólo económicos sino también sociales. Entre estos cabe destacar, por un lado, problemas asociados al juego en general, que incluyen conductas delictivas, como el fraude y la malversación, y también una mayor incidencia de suicidios, rupturas familiares, suspensiones de pagos, quiebras, etc.; y, por otro lado, problemas asociados al juego patológico, como el mayor riesgo de padecer alcoholismo, de cometer delitos relacionados con drogas o de violencia doméstica (Brunelle et al., 2012; Parker et al., 2013).

Los costes sociales del juego en menores y adolescentes se derivan del incremento en el riesgo de abandono o disminución del rendimiento escolar, el mayor nivel de ansiedad y depresión, aislamiento y violencia, junto con una mayor incidencia de conductas inapropiadas e incluso delictivas que se dan en los jugadores patológicos (Kuss y Griffiths, 2012; Derevensky y Gilbeau, 2015). Los menores tienden a no prestar atención a las normas de uso de las páginas web y menos aún al apartado de juego responsable; no hacen caso de las recomendaciones sobre no introducir datos 
personales o no mentir sobre su edad (Carbonell y Montiel, 2013). Por ello los problemas derivados de las ludopatías entre adolescentes y entre adultos deben ser tenidos en cuenta. Los Estados deben asumir el coste que se deriva de la incidencia de la ludopatía y deben establecer mecanismos que compensen el coste de los tratamientos y de los estudios de las consecuencias de la práctica de juegos de azar. La lógica impone que estos costes sean sufragados con parte de los beneficios que genera esta industria arbitrando mecanismos de reinversión, de forma que parte de los impuestos que la industria genera vayan a parar a las instituciones estatales de ayuda a los perjudicados.

Por otra parte, y como consecuencia de lo anterior, resulta necesario adoptar programas de prevención del desarrollo del juego entre los adolescentes con medidas eficaces para reducir los índices de juego patológico, adoptando iniciativas antes de la aparición de trastornos. Estas iniciativas para minimizar los daños causados por juegos de azar en Internet deben seguir las tres líneas básicas de aproximación a la prevención de daños: primaria, secundaria y terciaria. Las líneas primarias de intervención son aquellas que intentan proteger a las personas del daño antes de que este se desarrolle. Las intervenciones secundarias son aquellas que limitan el potencial del daño una vez que este ya existe. Las intervenciones terciarias son aquellas que intentan tratar aquellos que ya se encuentran seriamente afectados por el problema.

En las medidas de prevención deben tener parte activa los poderes públicos. Estos tienen la responsabilidad de regular la práctica del juego y controlar el cumplimiento de esta regulación. No todos los gobiernos lo han hecho y, en muchos casos, obvian aspectos importantes, como el acceso de los menores y otras personas vulnerables, la publicidad, el marketing y la implantación de medidas de juego responsable. Parece importante potenciar y financiar estudios sobre prevalencia y prevención en adultos y en menores. También se deben regular medidas de información sobre el juego que contrarresten la idea de aprobación y normalización en relación a los juegos de azar, especialmente entre los jóvenes y los adolescentes.

La Comisión Europea en su Libro Verde sobre el Juego online (2011) ha instado a las autoridades nacionales para que se aseguren de que todos los juegos ofertados estén sometidos en un control adecuado. La Comisión observa que la mayoría de los sitios web de juego online establecidos y autorizados en la Unión Europea contienen enlaces a servicios de ayuda $u$ organizaciones que trabajan con personas con problemas de juego. Sin embargo, pese a que quince Estados miembros han realizado estudios de prevalencia de ámbito nacional o regional, no existe apenas información sobre la oportuna respuesta de los poderes públicos ante tales estudios (p.ej. puesta en marcha de campañas educativas o recursos adicionales asignados a la prevención o el tratamiento), o sobre hasta qué punto las personas con problemas de juego tienen acceso a tratamientos. El Parlamento europeo ha instado a la Comisión a impulsar la formación de una red de organizaciones nacionales que se ocupen de los adictos al juego para hacer posible un intercambio de experiencias y prácticas acreditadas. La expresión de la "protección de las personas vulnerables", que incluso se ha introducido en la legislación inglesa (Rose, 2011: 69), hace referencia a aquellas personas que se pueden ver perjudicadas por el juego, razón por la cual el Estado debe protegerlas, como se protege a los niños. 
Entre las responsabilidades de las administraciones públicas en relación con los juegos de azar cabe mencionar las siguientes (Chóliz, 2008): controlar el acceso de menores y personas vulnerables, organizar los servicios sanitarios de ayuda a los afectados, coordinar las políticas de prevención en la sociedad en general, en escuelas y en empresas de juego, coordinar las políticas de información y de publicidad sobre la práctica del juego, ofrecer información detallada a los consumidores sobre la industria del juego, contrarrestando la idea de ganancias fáciles, controlar los mecanismos de pago sobre los intermediarios para asegurarse que no existen problemas de amaño, blanqueo de dinero, fraudes y acceso de menores y personas vulnerables. Asimismo, sería adecuado que fomenten la realización de estudios sobre el índice de prevalencia de juego patológico en menores y en adultos, y la difusión oportuna de los resultados de estos estudios.

\subsection{Prevención en la familia y en el colegio}

El medio más importante para la reducción de las consecuencias negativas asociadas al juego compulsivo es prevenir el problema antes de que aparezca. Dentro de la prevención primaria, además de las medidas legislativas para la reducción de la oferta de juego, son especialmente importantes las medidas educativas para la reducción de la demanda (Carpio, 2009: 40). En primer lugar, es fundamental la actitud que tienen los padres con los juegos de azar y que se den cuenta de que sus hijos aprenden las actitudes a través de ellos, pues son ellos los que primariamente deben transmitir una actitud saludable y equilibrada hacia la práctica de estas actividades. Se debe reforzar la idea de que perder tiempo y dinero, pedir dinero prestado o simplemente robar dinero para apostar son signos que demuestran que existe un problema con los juegos de azar entre los adolescentes ya que muchos de ellos no lo consideran así (Wiebe y FalkowskiHam, 2009).

Las fuentes que reconocen los adolescentes para informarse sobre salud son, principalmente, televisión, prensa, revistas, Internet y radio. También los profesionales sanitarios que acuden a los centros escolares y los propios profesores son considerados como voces fiables en materia de salud. La propia familia y los amigos de edades superiores completan el elenco de referencias informativas relevantes para los adolescentes (Coronado y López, 2006). Existen programas educativos para la puesta en marcha de programas de prevención. Algunos estudios (Carpio, 2009) demuestran que la información aumenta el conocimiento pero no es capaz de modificar los comportamientos, por lo que se necesitan otras estrategias de tipo educativo que aumenten las capacidades de los adolescentes en orden a defenderse de las consecuencias negativas del juego.

Entre las líneas de actuación que se han propuesto, cabe destacar las siguientes. En el contexto familiar, se debería disponer de información adecuada sobre las influencias de los medios de comunicación social y el fomento del juego, el proceso de adicción al juego, los efectos del juego (económicos, familiares, laborales, etc.), los factores de vulnerabilidad que desarrollan la adicción al juego. Es recomendable instaurar un estilo democrático de educación en el que existiese una comunicación fluida, normas claras y consensuadas, no excesivas, flexibles y adaptadas a cada edad y situación. 
Sería deseable evitar que los hijos entren en contacto con el juego a edades tempranas o enseñarles a valorarlo (por ejemplo: no permitirles manipular las máquinas o presionar el botón para iniciar la jugada, no darles monedas para jugar, etc.). Es importante, también, promocionar actitudes, valores y estilos de vida que sean incompatibles con las conductas de juego.

La escuela es un medio ideal para el desarrollo de los programas de intervención primaria que transmitan informaciones adecuadas y ayuden a desarrollar habilidades apropiadas para el control de la situación (Ladoucer, 1993: 370). Los objetivos generales de un programa educativo para la prevención del abuso del juego se podrían resumir en los siguientes (Carpio, 2009):

a) Informar a la población escolar sobre los riesgos que conlleva el juego y las características de la ludopatía.

b) Promocionar actitudes, valores y estilos de vida que sean incompatibles con las conductas de juego.

c) Desarrollar las habilidades necesarias para afrontar adecuadamente las situaciones de presión social asociadas con el juego, saber decir «no».

d) Fomentar actividades de ocio y tiempo libre incompatibles con el juego.

El objetivo de la educación en las escuelas sobre la problemática de los juegos de azar es hacer conscientes a los adolescentes de los riesgos y de los potenciales problemas asociados a los juegos de azar y apoyar a los estudiantes en las alternativas que existen. Los temarios incluyen los mitos, creencias, supersticiones y, también, información de lugares donde ir cuando existe un problema con el juego. También las estrategias para corregir los problemas de falta de conocimientos sobre lo que representa la probabilidad o la aleatoriedad deben destacar diferentes iniciativas desarrolladas en EE.UU. y en Inglaterra en las que diferentes problemas de matemáticas enseñan a los adolescentes a calcular los riesgos que pueden asumir en las apuestas (Wiebe y Falkowsky-Ham, 2003: 52). En este sentido las investigaciones llevadas a cabo por Shaffer (2003) revelaron una significativa relación inversa entre los estudiantes destacados en matemáticas y los juegos de azar. Los conocimientos de estadística y probabilidad pueden incrementar el interés en las matemáticas y también enseñar que los juegos de azar generalmente producen pérdidas. Ambas materias tienen el potencial de mejorar habilidades críticas en los estudiantes y de disminuir su interés en conductas potencialmente adictivas.

Las asociaciones cívicas y sociales pueden, por su parte, prestar apoyo psicológico a los afectados por la práctica del juego. De hecho, ofrecen teléfonos de consulta, establecen programas de tratamiento de la adicción, denuncian malas prácticas de publicidad o marketing, vigilan a las administraciones públicas y organizan foros o congresos relacionados con la práctica de los juegos de azar (The Allen Consulting Group, 2009). La intención de estas medidas es disminuir la proporción de personas que pueden desarrollar problemas con el juego y dar soporte a los jugadores problemáticos.

\subsection{Programas informativos y campañas de salud}

Dentro de las estrategias de minimización de daños, las campañas informativas ocupan un lugar relevante. Las estrategias de prevención dirigidas a los jóvenes requieren 
atención especial ya que muchas de las campañas de salud actuales se basan en el supuesto de que el conocimiento de los riesgos o peligros asociados a participar en una actividad vaya a traducirse en el rechazo de esa actividad. Sin embargo, los diseñadores de campañas tienden a ignorar el hecho de que las conductas prohibidas a menudo se ven bien entre los adolescentes (McMullan et al., 2012). Con el fin de que los adolescentes consideren los mensajes relacionados con la salud, los diseñadores de las campañas de prevención deben entender y respetar los puntos de vista de los adolescentes y explicar las consecuencias concretas de un comportamiento que son significativas para el grupo de personas al que se dirigen. Por ejemplo, los diseñadores de las campañas contra el tabaco han reconocido que las consecuencias negativas del mal aliento y dientes manchados eran mucho más importantes que las consecuencias de salud como el cáncer y las enfermedades del corazón. Los adolescentes perciben que los mensajes de los medios de comunicación sobre salud promueven escasamente comportamientos que modifiquen sus hábitos de salud. Y también consideran que la influencia de estos mensajes sobre la prevención de comportamientos de riesgo es insuficiente (Messerlian y Derevensky, 2007). Solo consideran que pueden influir estos mensajes cuando se presentan con realismo y con un formato atrayente para ellos (McMullan et al., 2012). También consideran los adolescentes que los mensajes sobre salud pueden ser negativos cuando son ambiguos por responder a intereses comerciales. El tratamiento que consideran adecuado en el caso de drogas, alcohol y tabaco es el tratamiento con requisitos realistas, claros y un lenguaje audiovisual relacionado con su mundo donde ellos se vean protagonistas y no haya manipulación por parte de los adultos (Coronado y López, 2006). La gran mayoría de los jóvenes han recibido los mensajes publicitarios, de la televisión o de Internet, sobre los juegos de azar y solo una minoría dice que ha visto mensajes que hablen de la responsabilidad en los juegos de azar (Wiebe y Falkowsky-Ham, 2003: 9). Este desequilibro sienta las bases de una comprensión sesgada de los impactos sobre los juegos de azar. Sin embargo, a pesar del generalizado acuerdo, se ha hecho relativamente poco para aplicar este aprendizaje en el área del problema de la prevención de los juegos de azar.

Las estrategias de prevención deben reforzar los problemas que se pueden crear como consecuencia de la práctica de los juegos de azar, que pongan de manifiesto argumentos que expliquen estas consecuencias, como pueden ser las peleas o amenazas por deudas de juego sin olvidar fórmulas que ayuden a evitar la presión de los compañeros para jugar. Las estrategias de comunicación deberían evitar mostrar los juegos de azar y las apuestas como una actividad divertida -que es como la perciben los adolescentes y propia de personas arriesgadas, populares y líderes (Wiebe y Falkowski-Ham, 2003)- y contrarrestar esa percepción con más información sobre las consecuencias sociales y personales del exceso en el juego.

\subsection{La responsabilidad de las empresas}

El concepto de Responsabilidad Social Corporativa pone énfasis sobre el papel de las empresas en la sociedad y la naturaleza de sus responsabilidades hacia el bienestar social. Las empresas cuya actividad comercial son los juegos de azar, son conscientes de que los juegos de azar por parte de los menores constituyen un problema y están 
preocupados por las consecuencias que esta actividad implica (Satre, 2003). Podemos resaltar aspectos importantes en relación a la responsabilidad de las empresas y los menores como son el hecho de que el juego de azar por parte de menores de edad es por definición ilegal, por lo que las empresas no están interesadas en que participen menores. Tomar parte en juegos de azar es una actividad percibida de forma parecida a comprar alcohol, conducir, votar o realizar un contrato. Se entiende que es necesaria una maduración emocional e intelectual para ejercer estas actividades antes de decidir involucrarse en ellas.

También es muy importante para las empresas de apuestas online cumplir con el marco legal: para los operadores y para los reguladores es imprescindible la buena reputación que deben tener frente a los consumidores y usuarios de la actividad y frente a la sociedad en general (Lamont et al., 2011: 20). Esta reputación se consigue cumpliendo con las características de la licencia. Deben controlar el acceso de las personas que juegan y deben controlar su actividad mientras juegan. Ello conlleva tener éxito comercial y al mismo tiempo asegura una buena recaudación de impuestos. Forma parte de los valores de las empresas de juego de azar no permitir jugar a menores. Existen empresas que implementan programas de juego responsable y son muy sensibles en la práctica correcta y eficaz del juego y se preocupan de formar a su personal para que pueda detectar a personas vulnerables o menores.

Los proveedores de los juegos de azar en Internet, por otra parte, deben establecer estrategias propias de minimización de daños para los jugadores vulnerables. Estas estrategias deben incluir información, como links de servicios de ayuda como servicios específicos online de ayuda que puedan asistir a los jugadores a la hora de limitar su juego. También aquellas estrategias que permiten que el jugador se autoexcluya, lo que implica que pueda cerrar una cuenta con facilidad, establecer límites en la cantidad de las apuestas, o en la cantidad de las pérdidas (The Allen Consulting Group, 2009). Todas estas acciones presuponen que el jugador reconoce que puede necesitar unos límites en su actividad de juego online.

Entre las medidas específicas de prevención de la adicción que se pueden promover desde las empresas, están políticas destinadas a desarrollar protocolos de buenas prácticas; dar información fidedigna, no generar falsas expectativas y generar información durante el proceso de juego online, por ejemplo entre apuesta y apuesta (Chóliz, 2013). También es importante el control de las variables estructurales de los juegos, como son no aceptar tarjetas de crédito, demorar las respuestas al menos diez segundos, limitar el importe de la apuesta y del gasto, dar indicaciones sobre cuánto dinero se ha perdido, no permitir acceso al crédito o establecer límites en las ganancias y en las pérdidas. (The Allen Consulting Group, 2009). Además, hay que tener en cuenta el control de las características ambientales y las políticas de marketing, pues la publicidad del juego online utiliza el mismo canal que sirve para jugar (Internet), operando con una inmediatez que no se da habitualmente en canales offline. Por eso, la publicidad es otro campo en el que es importante que se plasme la responsabilidad de las empresas (López Jiménez, 2013). El entorno es cada vez más competitivo y en este sentido la publicidad constituye un elemento diferenciador entre competidores pero no por ello deben ignorarse los límites que las regulaciones establecen. 


\section{El concepto de "juego responsable"}

El concepto de "juego responsable" surge en el contexto de las prácticas y principios adoptados por los gobiernos y otros gestores de juegos para mitigar los efectos negativos de los juegos de azar y proteger a las personas vulnerables (DGOJ, 2013). Los poderes públicos tienen la responsabilidad de poner los medios para evitar conductas patológicas en el juego, ser transparente en los recursos generados por el juego, utilizar publicidad no agresiva, establecer seguridad en la gestión, asegurar la integridad y la confidencialidad. Las políticas de juego responsable en España se encuentran establecidas en el art. 8 de la Ley de regulación del juego de 2011. Lamentablemente este artículo no ha sido desarrollado reglamentariamente, y ha quedado como una mera declaración de intenciones. En enero de 2015, el Ministerio de Hacienda y Administraciones Públicas, del que depende el Consejo Asesor de Juego Responsable, inauguró un sitio web (www. jugarbien.es) en el que se exhorta a "jugar con responsabilidad" y se ofrece información sobre riesgos, prevención y recursos para hacer frente al juego problemático.

Los objetivos deseables de un programa de "juego responsable" son la prevención, mediante la información y sensibilización, y la ayuda a los jugadores-problema para disminuir su práctica y afrontar las consecuencias negativas del exceso (Chóliz, 2013). La estrategia de "juego responsable" abarca diversas recomendaciones para los adultos aficionados a los juegos de azar: jugar con una frecuencia razonable -como una opción más dentro de la amplia oferta de entretenimiento- y en los espacios permitidos para ello; considerar que el objetivo final es la diversión o entretenimiento y no otros fines de carácter instrumental, como jugar para ganar dinero; antes de empezar a jugar definir un límite de dinero; jugar sólo con el dinero disponible; no utilizar dinero destinado a otros fines; no pedir dinero prestado para jugar; fijar un tiempo límite de juego en cada sesión y respetarlo; prever que el riesgo aumenta con las pérdidas; informarse acerca de las posibilidades de ganar y perder en cada juego; hacer pausas mientras se apuesta o juega, pues así se pueden reconsiderar las jugadas.

Se debe tener en cuenta que los intereses de los diferentes agentes que implica una estrategia de juego responsable no son coincidentes pues el límite de gasto para los jugadores es un límite de ingresos para las empresas. En efecto, la idea de "juego responsable" sugiere que el jugador realice un gasto razonable y se desincentive el juego compulsivo (Chóliz, 2010). Se busca explícitamente promover que los ciudadanos jueguen menos, lo cual tiene como consecuencia probable la reducción de los beneficios para las empresas del sector. Con todo, algunas empresas han emprendido unas acciones apropiadas sobre el juego responsable que implica el desarrollo de las siguientes acciones (Carpio, 2009): capacitar al personal sobre la noción de "juego responsable"; proporcionar a los clientes material gráfico en forma de folletos o en las webs con los conocimientos básicos sobre juego, juego inmoderado y autoexclusión voluntaria; ofrecer líneas de teléfono de información y orientación a jugadores o familiares en problemas; disponer de personal especializado para atender consultas en las webs de juego y ofrecer entrevistas de orientación; informar sobre sistemas de autoexclusión voluntaria a quien lo solicite; respetar la edad mínima de acceso, exigiéndola de forma visible y clara en las webs de juego, poniendo los medios técnicos posibles para procurar su cumplimiento. 
Existen guías, programas y campañas de prevención de riesgos en Internet, de diferentes asociaciones nacionales e internacionales (Carbonell y Montiel, 2013). Es una obligación de la industria del juego proporcionar sus servicios de forma que faciliten que el jugador pueda decidir responsablemente. También se incluyen avisos preventivos sobre la adicción en juegos ofertados por Internet, e incluso ofrecen test rápidos para detectar la gestación de problemas derivados de un juego no-responsable.

El "juego responsable" supone la necesidad de un equilibrio entre el marco normativo y la implicación del sector. La sostenibilidad es un factor de concienciación que debe ser complementario de otras medidas más directas como dedicar medios económicos relacionados con la recaudación pública a programas educativos, formativos, paliativos de aquellos efectos y de las secuelas que puede producir el juego. En este sentido sería interesante conocer la tasa de recursos destinados a la lucha contra las ludopatías de la recaudación fiscal por juego (por ejemplo, según el Informe del Defensor del Pueblo Andaluz, la tasa de recursos destinados a la lucha contra las ludopatías en 2002 era solo el $0,06 \%$ de la recaudación fiscal por juego). También cabe resaltar que las Apuestas Mutuas Deportivo Benéficas, disponen de un sistema muy pormenorizado de distribución de los ingresos fiscales, en el cual se contempla la cesión de un porcentaje a las Diputaciones provinciales para la construcción de equipamientos deportivos. Este es el orden de cosas que deberían inspirar el nuevo marco que se podría proponer (Gamero, 2006). En el mismo sentido también sería bueno constituir un fondo de asistencia para personas dependientes del juego, y a sus familiares, mediante un porcentaje de cada jugada de cualquier juego.

En cuanto a la protección de menores y de sectores vulnerables, todos los operadores serios y responsables deben utilizar sistemas y herramientas en sus web que ayuden en la detección y control de los clientes con posibilidades de desarrollar problemas con el juego. Estas herramientas incluyen límites de depósito y pérdidas, exclusión de productos, temporizadores, autoexclusión, preguntas de autoevaluación e información sobre organizaciones de ayuda. Algunos Estados requieren una "donación" de un porcentaje de beneficios a favor de una organización nacional de lucha contra la adicción del juego para ayudar en la financiación de sus servicios a los sectores vulnerables de la sociedad. Además, muchos operadores, como parte de sus programas de responsabilidad contribuyen a la investigación del juego responsable. El operador y el regulador deben tener las herramientas jurídicas y técnicas para detectar conductas anómalas de sus clientes a partir del momento en que cada cliente se registra en el sitio web. Es importante el establecimiento de mecanismos adecuados que monitoricen las operaciones y los grupos de riesgo. También son importantes las medidas anti fraude y los sistemas de certificación y homologación de equipos y de software, los requisitos de concesión de las licencias y el control del juego transfronterizo. En este sentido, las leyes tienen numerosos vacíos legales y su aplicación puede resultar problemática e ineficaz.

\section{Conclusiones}

En este artículo hemos expuesto la relación entre adolescentes y juegos de azar, cómo los perciben y por qué les resultan tan atractivos. La irrupción de los juegos de azar en 
Internet supone un riesgo de adicción para los adolescentes que se han acostumbrado a disfrutar de las ventajas de la red pero que no son capaces de entender sus riesgos. Algunos factores asociados particularmente al riesgo de exceso en el juego son la edad de inicio, la influencia de los padres, el consumo de sustancias estimulantes, la publicidad y las actitudes del grupo de iguales. El juego es visto, sobre todo, como una diversión emocionante, si bien la dificultad para diferenciar entre los conceptos de suerte, destino, azar y probabilidad, así como la familiaridad con videojuegos y otros juegos de ordenador convierte a los menores en consumidores vulnerables frente a los juegos de azar online.

Entre las consecuencias negativas que puede generar la participación en juegos de azar online, la más grave es el juego patológico, verdadero trastorno adictivo. Tanto los poderes públicos como la misma industria del juego deben considerar y concretar el desarrollo de planes integrales de juego responsable que garanticen un modelo de juego seguro y controlado. En este contexto, la publicidad y la promoción del juego debe tener en cuenta criterios de protección de los consumidores sabiendo que, aun cuando les está prohibido, los menores tienen facilidad de acceso al juego de azar online. Las Administraciones públicas deben establecer políticas de juego responsable y sensibilizar a la sociedad. Todos los agentes implicados en la actividad del juego deben por su parte aportar mecanismos de prevención y sensibilización hacia un problema del que existe aún poca conciencia social y una excesiva despreocupación.

\section{Bibliografía}

Aguaded-Gómez, J. (2011): "Niños y adolescentes: nuevas generaciones interactivas", Revista Comunicar, 18 (36), pp. 7-8.

Arbinaga, F. (2000): "Estudio descriptivo sobre el juego patológico en estudiantes (8-17 años): Características sociodemográficas, consumo de drogas y depresión", Adicciones, 1 (4), pp. 493-505.

Barnes, G., J. Welte, J. Hoffman, y M. Tidwell (2011): "The Co-occurrence of Gambling with Substance Use and Conduct Disorder among Youth in the United States", The American Journal on Addictions, 20 (2), pp. 166-173.

Becoña, E. (2004): "El juego patológico: prevalencia en España”, Salud y Drogas, 4 (2), pp. 9-34.

Becoña, E., M. Míguez y F. Vázquez (2001): "El juego problema en los estudiantes de Enseñanza Secundaria", Psicothema, 13 (4) pp. 551-556.

Brunelle, N., D. Leclerc, M. Cousineau, M. Dufour, A. Gendron e I. Martin (2012): "Internet gambling, substance use, and delinquent behavior: An adolescent deviant behavior involvement pattern", Psychology of addictive behaviors, 26 (2), pp. 364370.

Carbonell, E. e I. Montiel (eds.) (2013): El juego de azar online en los nativos digitales, Valencia, Tirant Humanidades.

Carpio, C. (2009): "Aspectos psicológicos del juego comercial. Tratamientos y programas preventivos. Hacia el juego responsable", Docencia e Investigación: Revista de la Escuela Universitaria de Magisterio de Toledo, 34 (19) pp. 25-58. 
Chóliz, M. (2008): Adicción al juego de azar, Buenos Aires, deauno.com.

Chóliz, M. (2010): "Experimental analysis of the game in pathological gamblers: Effect of the immediacy of the reward in slot machines", Journal of Gambling Studies, 26, pp. 249-256.

Chóliz, M. (2013): "Una propuesta de juego responsable en la situación española actual", Revista Infocop Online. Disponible en:

http://www.cop.es/infocop/pdf/2201.pdf.

Comisión Europea (2011): Libro verde sobre el juego en línea en el mercado interior. Disponible en:

http://eurlex.europa.eu/LexUriServ/LexUriServ.

do?uri=COM:2011:0128:FIN:ES:PDF (Consulta: 12 de noviembre de 2015)

Comisión Europea (2014): Recommendation on principles for the protection of consumers and players of online gambling services and for the prevention of minors from gambling online web. Disponible en:

http://ec.europa.eu/internal_market/gambling/docs/initiatives/140714-commissionrecommendation-on-online-gambling_en.pdf (Consulta: 12 de noviembre de 2015)

Coronado, J. M. y J. M. López (2006): “Comunicación y salud pública desde los adolescentes", Revista Comunicar, 13 (26), pp. 63-71.

Cosenza, M. y G. Nigro (2015): "Wagering the future: Cognitive distortions, impulsivity, delay discounting, and time perspective in adolescent gambling", Journal of Adolescence, 45, pp. 56-66.

Derevensky, J. L. (2012): Teen gambling: Understanding a growing epidemic, Londres, Rowman \& Littlefield Publishers.

Derevensky, J. L., y L. Gilbeau (2015): “Adolescent Gambling: Twenty-five Years of Research", Canadian Journal of Addiction, 6 (2), pp. 4-12.

Derevensky, J. L. y R. Gupta (2005): Gambling problems in youth, Nueva York, Springer.

Derevensky, J. L. y R. Gupta (2007): 'Internet gambling amongst adolescents: A growing concern", International Journal of Mental Health and Addiction, 5 (2), pp. 93-101.

Derevensky, J. L., R. Gupta y K. Winters (2003): "Prevalence rates of youth gambling problems: Are the current rates inflated?", Journal of Gambling Studies, 19 (4), pp. 405-425.

Derevensky, J. L., C. Temcheff, y R. Gupta (2011): "Treatment of adolescent gambling problems: More art than science?", en J. L. Derevensky (ed.), Youth Gambling: The Hidden Addiction, Berlin, De Gruyter, pp. 167-186.

DGOJ. Dirección General de Ordenación del Juego (2013): Estrategia del Juego Responsable en España, Disponible en: http://www.ordenacionjuego.es/es/estrategia-juego-responsable .

Domínguez, A. M. (2007): Guía Clínica: Actuar en el juego patológico, Dirección General para las Drogodependencias y Adicciones, Consejería para la Igualdad y Bienestar Social, Junta de Andalucía. 
Donati, M., F. Chiesi, y C. Primi (2013): “A model to explain at-risk/problem gambling among male and female adolescents: Gender similarities and differences", Journal of Adolescence, 36 (1), pp. 129-137.

Dorrego de Carlos, A. (2011): "E-Gambling: un nuevo escenario jurídico de la Sociedad Global", en J. Cases Méndez y Gusano Serrano, eds., Nuevos Escenarios en el Juego de Azar: La Globalización, Cizur Menor, Aranzadi Thomson-Reuters, pp. 15-52.

Duek, C. (2010): "Infancia, medios de comunicación y juego: Un campo de trabajo, un objeto de estudio", Iberoamerica Global, 3 (1), pp. 53-74.

Erbas, B. y U. Buchner (2012): "Pathological Gambling Prevalence, diagnosis, comorbidity, and intervention in Germany", Deutscher Arzteblatt International, 109 (10), pp. 173-182.

EU.net (2012): Investigación sobre conductas adictivas a Internet entre los adolescentes europeos. Disponible en: http://www.protegeles.com/docs/estudio_conductas_internet.pdf.

Felsher, J. R., J. L. Derevensky y R. Gupta (2010): "Young adults with gambling problems: The impact of childhood maltreatment", International Journal of Mental Health and Addiction, 8 (4), pp. 545-556.

Fernández, J. (2009): “Comprar la madurez”, en X. Bringué y C. Sádaba, eds., Nacidos Digitales: Una generación frente a las pantallas, Pamplona, Rialp, pp. 169-191.

Floros, G., K. Siomos, V. Fisoun y D. Geroukalis (2013): "Adolescent online gambling: The impact of parental practices and correlates with online activities", Journal of Gambling Studies, 29 (1), pp. 131-150.

Fröberg, F. (2006): Gambling among young people, a knowledge review, Estocolmo: Swedish National Institute of Public Health.

Gamero, E. (2004): "Limitaciones personales a la libertad de jugar: régimen jurídicoadministrativo del jugador", en P. Olmeda (ed.), Régimen del Juego en España, Cizur Menor: Aranzadi Thomson-Reuters, pp. 741-768.

Gendron, A., N. Brunelle, D. Leclerc, M. Dufour y M. Cousineau (2009): "Comparison of the profiles of young non-gamblers, gamblers and Internet gamblers relative to psychological distress, severity of substances use and impulsiveness/risk taking", en 8th Annual Conference Alberta Gaming Resources Institute, Banff Center, Alberta.

Gómez Yañez, J. A., J. I. Cases Méndez, C. Lalanda y G. Gusano (2015): Percepción social sobre el juego de azar en España (V), Madrid, Instituto de Política y Gobernanza, Universidad Carlos III. Disponible en:

http://www.codere.com/uploads/media/ default/0001/03/6424715e849f43016ad3dbb9775bcb5a25113e1e.pdf.

Granero, R., E. Penelo, R. Stinchfield, F. Fernández-Aranda, L. Savvidou, F. Fröberg, N. Aymamí, M. Gómez-Peña, M. Pérez-Serrano, A. del Pino-Gutierrez, J. M. Menchón y S. Jiménez-Murcia (2014): "Is pathological gambling moderated by age?", Journal of Gambling Studies, 30 (2), pp. 475-492.

Griffiths, M. (2001): "Internet gambling: Preliminary results of the first UK prevalence study." Journal of Gambling Issues, 5.

Griffiths, M. (2002): Gambling and Gaming Addictions in Adolescence. Pacts 2. Parent, Adolescence and Training Skills, Londres, Blackwell. 
Griffiths, M. (2009): Problem gambling in Europe: an Overview. Disponible en: http://www.nsgamingfoundation.org/uploads/Problem\%20Gambling\%20in\%20 Europe.pdf

Griffiths, M. y J. Parke (2010): “Adolescent gambling on the Internet: A review", International Journal of Adolescent Medicine and Health, 22 (1), pp. 59-75.

Griffiths, M., H. Wardle, J. Orford, K. Sproston y B. Erens (2009): "Sociodemographic correlates of internet gambling: Findings from the 2007 British Gambling Prevalence Survey", CyberPsychology \& Behavior, 12 (2), pp. 199-202.

Griffiths, M. y R. Wood (2000): "Risk factors in adolescence: The case of gambling, videogame playing, and the Internet", Journal of Gambling Studies, 16 (2-3), pp. 199-225.

Gupta, R. y J. L. Derevensky (2014): "Reflections of underage gambling”, Responsible Gambling Review, 1 (1), pp. 37-50.

Hardoon, K., J. L. Derevensky y R. Gupta (2003): "Empirical measures vs. perceived gambling severity among youth: why adolescent problem gamblers fail to seek treatment", Addictive Behaviors, 28 (5), pp. 933-946.

Ipsos MORI (2009): British survey of Children, the National lottery and Gambling 2008-2009. Report of a quantitative survey, Londres, National Lottery Comission.

Jiménez-Murcia, S., F. Fernández-Aranda, R. Granero y J. M. Menchón (2014): "Gambling in Spain: update on experience, research and policy", Addiction, 109 (10), pp. 1595-1601.

King, D. L., P. Delfabbro, D. Kaptsis y T. Zwaans (2014): "Adolescent simulated gambling via digital and social media: An emerging problem", Computers in Human Behavior, 31, pp. 305-313.

Kopp, J. (2015): "Un estudio del juego entre los jugadores de juegos de rol masivos en línea (MMORPG)", Lúdicamente, 4 (7).

Kuss, D. y M. Griffiths (2012): "Internet gaming addiction: A systematic review of empirical research", International Journal of Mental Health and Addiction, 10 (2), pp. 278-296.

Ladoucer, R. (1993): “Aspectos fundamentales y clínicos de la psicología de los juegos de azar y de dinero", Psicología conductual, 1, pp. 361-374.

Lamont, M., N. Hing y S. Gainsbury (2011): "Gambling on sport sponsorship: A conceptual framework for research and regulatory review", Sport Management Review, 14, pp. 247-257.

Lesieur, H. (2003): “Adolescent gambling research. The next wave”, en H. Shaffer, M. Hall, J. Vander Bilt y E. George, (eds.), Youth, gambling \& society: Futures at stake, Reno, University of Nevada Press, pp. 228-245.

Livingstone, S. y B. Moira, (eds.) (2013): Children and their changing media environment: A European comparative study, Londres, Routledge.

López Jiménez, D. (2013): "La regulación y la autorregulación en la publicidad, patrocinio y actividades asociadas al juego on line", en P. Olmeda, ed., En torno al juego de azar. Actividad, regulación y actores, Cizur Menor, Thomson ReutersAranzadi, pp. 153-268. 
McBride, J. y J. L. Derevensky (2012): "Internet gambling and risk-taking among students: An exploratory study", Journal of Behavioral Addictions, 1 (2), pp, 50-58.

McMullan, J., D. Miller y D. Perrier (2012): "I've Seen Them So Much They Are Just There: Exploring Young People's Perceptions of Gambling in Advertising", International Journal Mental Health Addiction, 10, pp. 829-848.

Messerlian, C., A. Byrne y J. L. Derevensky (2004): "Gambling, youth and the internet: should we be concerned?", The Canadian Child and Adolescent Psychiatry Review, 13 (1), pp. 3-12.

Messerlian, C. y J. L. Derevensky (2007): "Evaluating the role of social maketing campaigns to prevent youth gambling problems", Canadian Journal of Public Health, 98 (2), pp. 101-104.

Messerlian, C., J. L. Derevensky y R. Gupta (2005): "Youth gambling problems: a public health perspective", Journal of Gambling Issues, 14.

Muñoz-Molina, Y. (2008): "Meta-análisis sobre juego patológico, 1997-2007”. Revista de Salud Pública, 10 (1), pp. 150-159.

Olason, D., E. Kristjansdottir, H. Einarsdottir, H. Haraldsson, G. Bjarnason y J.L. Derevensky (2011): "Internet gambling and problem gambling among 13 to 18 year old adolescents in Iceland", International Journal of Mental Health and Addiction, 9 (3), pp. 257-263.

Organización Mundial de la Salud (1995): Clasificación Estadística Internacional de Enfermedades y Problemas relacionados con la Salud. Décima revisión CIE-10. Ginebra.

Parker, J., L. Summerfeldt, R. Taylor, P. Kloosterman, K. Keefer (2013): “Problem gambling, gaming and Internet use in adolescents: Relationships with emotional intelligence in clinical and special needs samples", Personality and Individual Differences, 55, pp. 288-293.

Petit, A., L. Karila, M. Lejoyelux (2015): "Le jeu pathologique chez l'adolescent", Archives de pédiatrie, 22 (5) p. 564-568.

Raisamo, S., J. Halme, A. Murto y T. Lintonen (2013): "Gambling-related harms among adolescents: a population-based study", Journal of Gambling Studies, 29 (1), pp. 151-159.

Rose, I. (2011): "El Juego On Line”, en Cases Méndez, J.I. y Gusano Serrano (eds.), Nuevos Escenarios en el Juego de Azar: La Globalización, Cizur Menor, Aranzadi Thomson-Reuters, pp. 53-80.

Ruiz-Olivares, R., V. Lucena, M. J. Pino y J. Herruzo (2010): “Análisis de comportamientos relacionados con el uso/abuso de Internet, teléfono móvil, compras y juego en estudiantes universitarios", Adicciones, 27 (4), pp. 301-310.

Satre, P. (2003): "Youth gambling. The casino industry's response", en H. Shaffer, M. Hall, J. Vander Bilt y E. George (eds.), Youth, gambling \& society: Futures at stake, Reno, University of Nevada Press, pp. 199-207.

Scholes-Balog, K., S. Hemphill, N. Dowling y J. W. Toumbourou (2014): “A prospective study of adolescent risk and protective factors for problem gambling among young adults", Journal of Adolescence, 37, pp. 215-224. 
Shaffer H. (2003): "The emergence of gambling among youth", en H. Shaffer, M. Hall, J. Vander Bilt y E. George (eds.), Youth, gambling \& society: Futures at stake, Reno, University of Nevada Press, pp. 25-38.

Shead, N., J. L. Derevensky, T. Fong, y R. Gupta (2012): “Characteristics of Internet gamblers among a sample of students at a large, public university in Southwestern United States", Journal of College Student Development, 53 (1), pp. 133-148.

Splevins, K., S. Mireskandari, K. Clayton y A. Blaszczynski (2010): "Prevalence of Adolescent Problem Gambling, Related Harms and Help-Seeking Behaviours Among an Australian Population", Journal of Gambling Studies, 26 (2), pp. 189204.

Svensson, O. (2011): "Gambling: Electronic friends or a threat to one's health and personal development?", International Journal of Qualitative Studies on Health and Well-being, 6 (2).

The Allen Consulting Group (2009): Review of current and future trends in interactive gambling activity and regulation, Disponible en: https://www.dss.gov.au/sites/default/files/documents/05_2012/gambling_25june. pdf

Tsitsika, A., E. Critselis, M. Janikian, G. Kormas y D. Kafetzis (2011): "Association Between Internet Gambling and Problematic Internet Use Among Adolescents", Journal of Gambling Studies, 27, pp. 389-400.

Valentine, G. (2008): Literature review of children and young people's gambling, Gambling Commission UK, Birmingham.

Vander Bilt, J. y J. Franklin (2003): "Gambling in a Familiar Context", en H. Shaffer, M. Hall, J. Vander Bilt y E. George (eds.), Youth, gambling \& society: Futures at stake, Reno, University of Nevada Press, pp. 100-124.

Volberg, R., R. Gupta, M. Griffiths, D. Olason y P. Delfabbro (2010): “An international perspective on youth gambling prevalence studies", International Journal of Adolescent Medicine and Health, 22 (1), pp. 3-38.

Weinberger, A., C. Franco, R. Hoff, C. Pilver, M. Steinberg, L. Rugle y M. Potenza (2015): "Gambling behaviors and attitudes in adolescent high-school students: Relationships with problem-gambling severity and smoking status", Journal of Psychiatric Research, 65, pp. 131-138.

Welte, J., G. Barnes, M. Tidwell y J. Hoffman (2008): "The prevalence of problem gambling among US adolescents and young adults: Results from a national survey", Journal of Gambling Studies, 24 (2), pp. 119-133.

Wiebe, J. y A. Falkowsky-Ham (2003): Understanding the Audience: The Key to Preventing Youth Gambling Problems. Disponible en:

http:/citeseerx.ist.psu.edu/viewdoc/download?doi=10.1.1.204.7918\&rep=rep1\&type=pdf.

Wood, R. T. y R. Williams (2009): Internet Gambling: Prevalence, Pattern, Problems and Policy Options, Final Report prepared for the Ontario Problem Gambling Research Centre, Guelph, Ontario. Disponible en:

https://www.uleth.ca/dspace/bitstream/handle/10133/693/2009-InternetPPPP-

OPGRC.pdf? sequence=4. 